\title{
Reverse transcriptases of retroviruses and retroelements: an evolutionary perspective Irina R Arkhipova
}

\author{
Address: Josephine Bay Paul Center for Comparative Molecular Biology and Evolution, Marine Biological Laboratory, 7 MBL Street, Woods Hole, \\ MA 02543, USA \\ from Frontiers of Retrovirology: Complex retroviruses, retroelements and their hosts \\ Montpellier, France. 21-23 September 2009
}

Published: 24 September 2009

Retrovirology 2009, 6(Suppl 2):O2 doi:10.1186/1742-4690-6-S2-O2

This abstract is available from: http://www.retrovirology.com/content/6/S2/O2

(c) 2009 Arkhipova; licensee BioMed Central Ltd.

Reverse transcriptases (RT) are central to retrovirology (as the key enzymes encoded by retroviruses) as well as to evolutionary genomics (as the only unifying component for numerous types of retroelements inhabiting genomic DNA in both prokaryotes and eukaryotes). A great deal of effort has been put into understanding the evolutionary relationships between RT-containing entities, including retroviruses, retrotransposons, and reverse transcriptaserelated genes such as telomerases. However, there is still no consensus on phylogenetic history of retroelements [1$3]$. The recent explosion in the amounts of genome sequence data from evolutionarily diverse organisms provides us with a rich source of novel RT-related sequences, which may be regarded as evolutionary intermediates and "missing links" connecting seemingly unrelated groups of reverse transcriptases. Our genomic surveys of rotifers of the Class Bdelloidea and comparisons with data from other sequenced genomes provided us with a number of examples of novel RT sequences fitting this description, including both published [3-5] and unpublished findings. Structure-based alignments and comparisons of RTassociated $\mathrm{N}$ - and C-terminal extension domains in newly identified groups of retroelements reveal deeper evolutionary connections between different RT classes than previously uncovered by phylogenetic analyses of the core RT domain spanning the seven most highly conserved motifs. Reverse transcriptase-related genes appear to be more common than previously thought, and may have independently given rise to mobile genetic elements several times in early evolution, via subsequent association with various catalytic domains able to cleave target genomic DNA and thereby confer intragenomic mobility to retroelement-encoded RNAs.

\section{References}

I. Malik HS, Eickbush TH: Phylogenetic analysis of ribonuclease $\mathbf{H}$ domains suggests a late, chimeric origin of LTR retrotransposable elements and retroviruses. Genome Res 200I, II:II87-II 197.

2. Chang GS, Hong Y, Ko KD, Bhardwaj G, Holmes EC, Patterson RL, van Rossum DB: Phylogenetic profiles reveal evolutionary relationships within the "twilight zone" of sequence similarity. Proc Natl Acad Sci USA 2008, I 05: I 3474- I 3479.

3. Arkhipova IR, Pyatkov KI, Meselson M, Evgen'ev MB: Retroelements containing introns in diverse invertebrate taxa. Nature Genetics 2003, 33: 123-124.

4. Gladyshev EA, Arkhipova IR: Telomere-associated endonuclease-deficient Penelope-like retroelements in diverse eukaryotes. Proc Natl Acad Sci USA 2007, 104:9352-9357.

5. Gladyshev EA, Meselson M, Arkhipova IR: Massive horizontal gene transfer in bdelloid rotifers. Science 2008, 320:1210-12/3. 